\title{
Freeze Drying Improves the Shelf-Life of Conductive Polymer Modified Neural Electrodes
}

\author{
Himadri S. Mandal ${ }^{1, *}$, Richard O. Cliff ${ }^{2}$ and Joseph J. Pancrazio ${ }^{3}$ \\ 1 Blackrock Microsystems, 630 Komas Dr \#200, Salt Lake City, UT 84108, USA \\ 2 System of Systems Analytics, 11250 Waples Mill Road, Fairfax, VA 22030, USA; \\ E-Mail: rcliff@sosacorp.com \\ 3 Department of Bioengineering, Volgenau School of Engineering, George Mason University, \\ 4400 University Drive, MS 1G5, Fairfax, VA 22030, USA; E-Mail: jpancraz@gmu.edu \\ * Author to whom correspondence should be addressed; E-Mail: hmandal@blackrockmicro.com; \\ Tel.: +1-801-582-5533; Fax: +1-801-582-1509.
}

Academic Editor: Anthony Guiseppi-Elie

Received: 29 June 2015 / Accepted: 4 August 2015 / Published: 7 August 2015

\begin{abstract}
Coating microelectrodes with conductive polymer is widely recognized to decrease impedance and improve performance of implantable neural devices during recording and stimulation. A concern for wide-spread use of this approach is shelf-life, i.e., the electrochemical stability of the coated microelectrodes prior to use. In this work, we investigated the possibility of using the freeze-drying process in order to retain the native low impedance state and, thereby, improve the shelf-life of conductive polymer poly(3,4-ethylenedioxythiophene) (PEDOT)-PSS modified neural electrodes. Control PEDOT-PSS coated microelectrodes demonstrated a significant increase in impedance at $1 \mathrm{kHz}$ after 41-50 days of room temperature storage. Based on equivalent circuit modeling derived from electrochemical impedance spectroscopy, this increase in impedance could be largely attributed to a decrease in the interfacial capacitance consistent with a collapse and closing of the porous structure of the polymeric coating. Time-dependent electrochemical impedance measurements revealed higher stability of the freeze-dried coated microelectrodes compared to the controls, such that impedance values after 41-50 days appeared to be indistinguishable from the initial levels. This suggests that freeze drying PEDOT-PSS coated microelectrodes correlates with enhanced electrochemical stability during shelf storage.
\end{abstract}


Keywords: conductive polymer; PEDOT; microwires; impedance; neural electrode

\section{Introduction}

Conductive polymer (CP) modified microelectrodes are promising for developing small but sensitive and powerful neural interfaces for recording and stimulation [1-8]. Because of the porous morphology, $\mathrm{CP}$ modified electrodes offer a high degree of electrochemically active surface area and have orders of magnitude lower impedance compared to their metallic counterparts [9,10]. Poly(3,4ethylenedioxythiophene) (PEDOT), more specifically PEDOT-PSS (Poly(sodium 4-styrenesulfonate)) (Figure 1), is the most extensively studied CP that has been used to modify neural microelectrodes [3,11-14].

In general, the PEDOT modification of neural microelectrodes is very straightforward and involves electrochemical deposition [3,11-14]. But a major consideration related to the handling of these modified microelectrodes is the concern that they may not retain their low impedance state under ambient conditions. For this reason, PEDOT electrodes are prepared as needed and quickly used during experimentation. This short shelf-life leads to a severe limitation in terms of storage and/or transportation, and is a bottleneck for a wide spread use of the polymer modified microelectrodes. To date, there is no report in the literature that addresses this issue.

In our previous reports $[7,8]$ on the mechanistic investigation of the solution aging process in PEDOT modified neural electrodes, we demonstrated a direct relationship between the time-dependent increase in the electrode impedance and the gradual decrease in the electrochemically active surface area due to the collapse of the porous polymeric morphology. Based on these observations, we suspected that a similar type of mechanism could also be involved behind the low shelf-life of PEDOT modified electrodes. In addition, based on some reports describing the "capillary-driven" collapse of porous nano-materials after solvent evaporations [15-18], we hypothesized that the drying process after the solution phase electrochemical PEDOT modification is probably a critical step that requires a thorough investigation.

Freeze-drying, also known as lyophilization, involves the removal of the solvent directly from the solid phase to the vapor phase and has been reported to keep the native structure intact in porous nano-materials $[19,20]$. Inspired by these findings, we postulated that the capillary-driven collapse could be avoided by freeze-drying PEDOT modified neural electrodes after the electrodeposition. In this work, we show that freeze-drying can indeed significantly increase the stability of PEDOT modified electrodes.

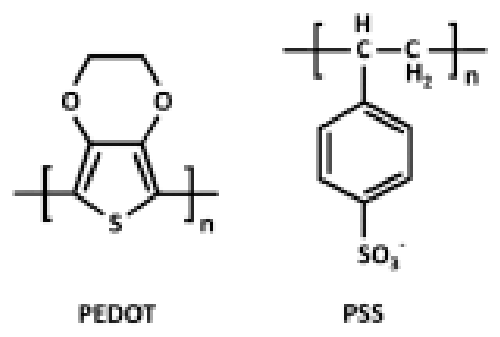

Figure 1. Chemical structures of Poly(3,4-ethylenedioxythiophene) (PEDOT) and Poly(sodium 4-styrenesulfonate) (PSS). 


\section{Experimental Section}

\subsection{Materials}

3,4-Ethylenedioxylthiophene (EDOT) and Poly(sodium 4-styrenesulfonate) (PSS) were purchased from Sigma-Aldrich (St. Louis, MO, USA). Phosphate-buffered saline (PBS) was from Mediatech Inc. (Manassas, VA, USA). Pt/Ir implantable microwires (PI20030.1A5) were obtained from MicroProbes for Life Sciences (Gaithersburg, MD, USA).

\subsection{Preparation of Polymer Modified Implantable Microwires}

Cyclic voltammetry (CV) was used to dynamically eletrodeposit [21] the polymer (PEDOT:PSS) on $\mathrm{Pt} / \mathrm{Ir}$ implantable microwires using a CHI 660D potentiostat (CH Instruments, Inc., Austin, TX, USA) from an aqueous solution $(50 \mathrm{~mL})$ of EDOT $(0.01 \mathrm{M})$ with $0.1 \mathrm{M}$ PSS. The potential range was $0-0.75 \mathrm{~V}$ vs. $\mathrm{Ag} / \mathrm{AgCl}$ reference electrode (scan rate was $1 \mathrm{~V} / \mathrm{s} ; 250$ cycles, total charge $3.5 \mu \mathrm{C}$ ).

\subsection{Freeze-Drying of Polymer Modified Implantable Microwires}

After the PEDOT modification, the microwire electrodes were rinsed with PBS, EIS was recorded and quickly freeze-dried using a Virtis Advantage (Gardiner, NY) lyophilizer. The lyophilization protocol was immediate shelf freezing to $-40{ }^{\circ} \mathrm{C}$, followed by $12 \mathrm{~h}$ of primary drying, with subsequent temperature ramping to $20{ }^{\circ} \mathrm{C}$ and 8 additional hours for secondary drying. The freeze-dried microwires were placed in individual vials, flushed with high purity Nitrogen gas, sealed and stored. The control non-freeze-dried PEDOT: PSS modified microwires were similarly flushed with Nitrogen and stored.

\subsection{Cyclic Voltammetry (CV), Electrochemical Impedance Spectroscopy (EIS) and Modeling}

$\mathrm{CV}$ and EIS of the modified Pt/Ir microwires were recorded using a potentiostat (CHI 660D, $\mathrm{CH}$ Instruments, Inc., Austin, TX, USA) in PBS at $22{ }^{\circ} \mathrm{C}$. The potential range in CV was $0-0.5 \mathrm{~V} v s$. $\mathrm{Ag} / \mathrm{AgCl}$ reference electrode (scan rate was $1 \mathrm{~V} / \mathrm{s}$ ). The impedance was measured at the open circuit potential from $0.1 \mathrm{~Hz}$ to $1 \mathrm{MHz}$, with a peak-to-peak amplitude of the sinusoidal voltage of $20 \mathrm{mV}$. ZsimpWin (Princeton Applied Research, Oak Ridge, TN, USA) was used to fit the EIS data.

\section{Results and Discussion}

A total of 50 commercially available implantable $\mathrm{Pt} / \mathrm{Ir}$ microwire electrodes were electrochemically modified with PEDOT-PSS. Figure $2 b$ shows the typical impedance of a microwire in PBS before and after the PEDOT modification. The impedance decreased over a wide a range of frequency and the corresponding non-Faradic current in CV (Figure 2c) increased significantly upon the modification which are in line with previous publications, and are related to the high surface area due to porous polymeric morphology [5,6,12,22-24]. 25 microwire electrodes were freeze-dried, stored and sealed under $\mathrm{N}_{2}$ in individual vials. The control group of 25 non-freeze-dried PEDOT-PSS modified microwires were similarly flushed with $\mathrm{N}_{2}$ and stored. 
(a)

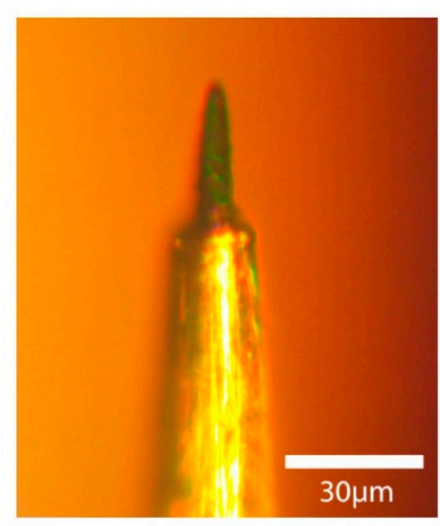

(b)

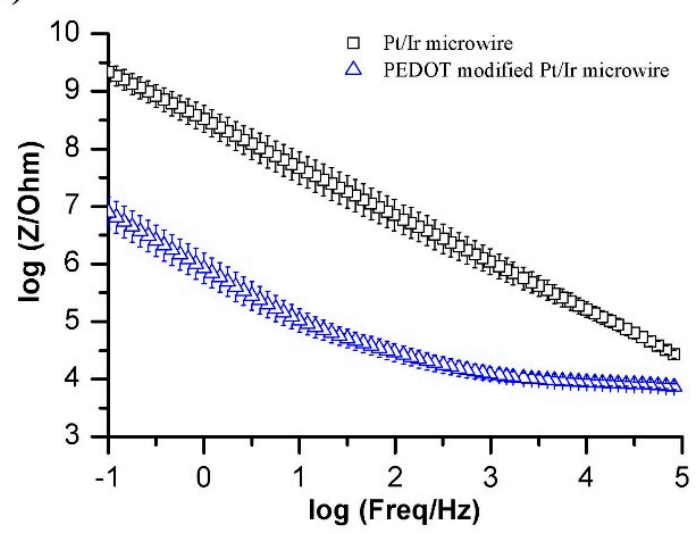

(c)

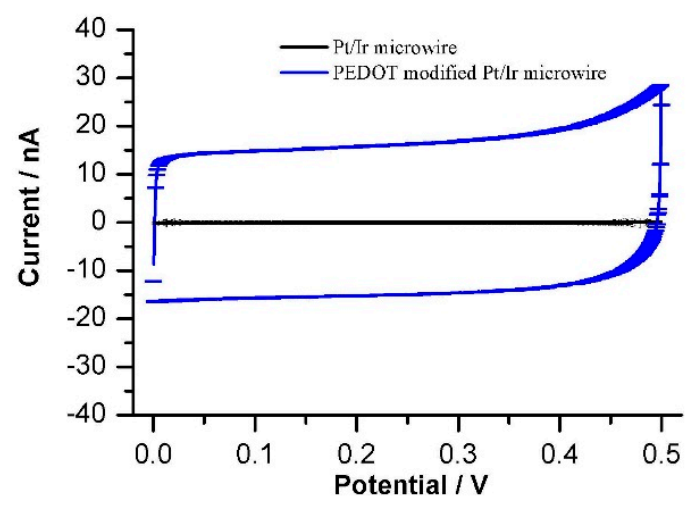

Figure 2. (a) Optical image of a PEDOT-PSS modified Pt/Ir microwire electrode; (b) Electrochemical Impedance Spectroscopy (EIS) and (c) Cyclic voltammetry (CV) (with the coefficient of variation) of microwires in phosphate-buffered saline (PBS) before and after the PEDOT-PSS modification.

Every 10 days, 5 freeze-dried PEDOT modified microwires and 5 controls were taken out from sealed vials, placed in PBS, and EIS was recorded. Any electrode showing 10\% increase in the impedance at $1 \mathrm{kHz}$ compared to that immediately after electrochemical deposition was considered to be failed. The impedance magnitude at $1 \mathrm{kHz}$ was chosen because of its correspondence to the bandwidth of common electrophysiologic signals associated with neural recording [1,2,12,22]. Freeze-dried PEDOT modified microwires showed higher stability compared to the non-freeze-dried controls (Figure 3f). Note that we also recorded the $\mathrm{CV}$ of the PEDOT modified electrodes (Figure S1). However, the change in the non-Faradic current in $\mathrm{CV}$ of the corresponding failed electrodes was found to be negligible most probably because of the insensitivity of $\mathrm{CV}$ in the $1 \mathrm{kHz}$ regime.

In order to reveal the failure mechanism in the non-freeze-dried PEDOT modified microwires, we fit the EIS data for a failed device (d41-d50) using a previously published [7] electrical equivalent circuit model for CP modified implantable neural microelectrodes (Figure $3 \mathrm{e}$ ). Here, $\mathrm{R}_{\text {poly }}$ and $\mathrm{Q}_{\text {poly }}$ are the charge transfer resistance and the constant phase element or CPE of the associated non-ideal double layer capacitor across the polymeric interface, respectively. $\mathrm{R}_{\mathrm{S}}$ is the solution resistance and $\mathrm{C}_{\mathrm{d}}$ is the double layer capacitance due to the pores and/or defect sites that directly expose the metallic electrode surface to the solution. $\mathrm{R}_{\text {poly }}$ remains similar (Table 1) indicating that the inherent polymer conductivity is not significantly affected which is further supported by the unchanged corresponding 
Qpoly. This is in contrast to that observed in solution aging process where we observed a significant increase in the $\mathrm{R}_{\text {poly }}$ indicating a time dependent polymeric aging [7,8]. However, $\mathrm{C}_{\mathrm{d}}$ decreases by orders of magnitude for the failed microwire and points to a reduction in the electrochemical surface area. This is consistent with the previously published solution aging process [7,8] and most likely specifies the increase in the impedance due to the collapse and closing of the porous structure. The increase in impedance in the high frequency regime (Figure 2d) may indicate closing of the relatively smaller pores of the polymer structure.
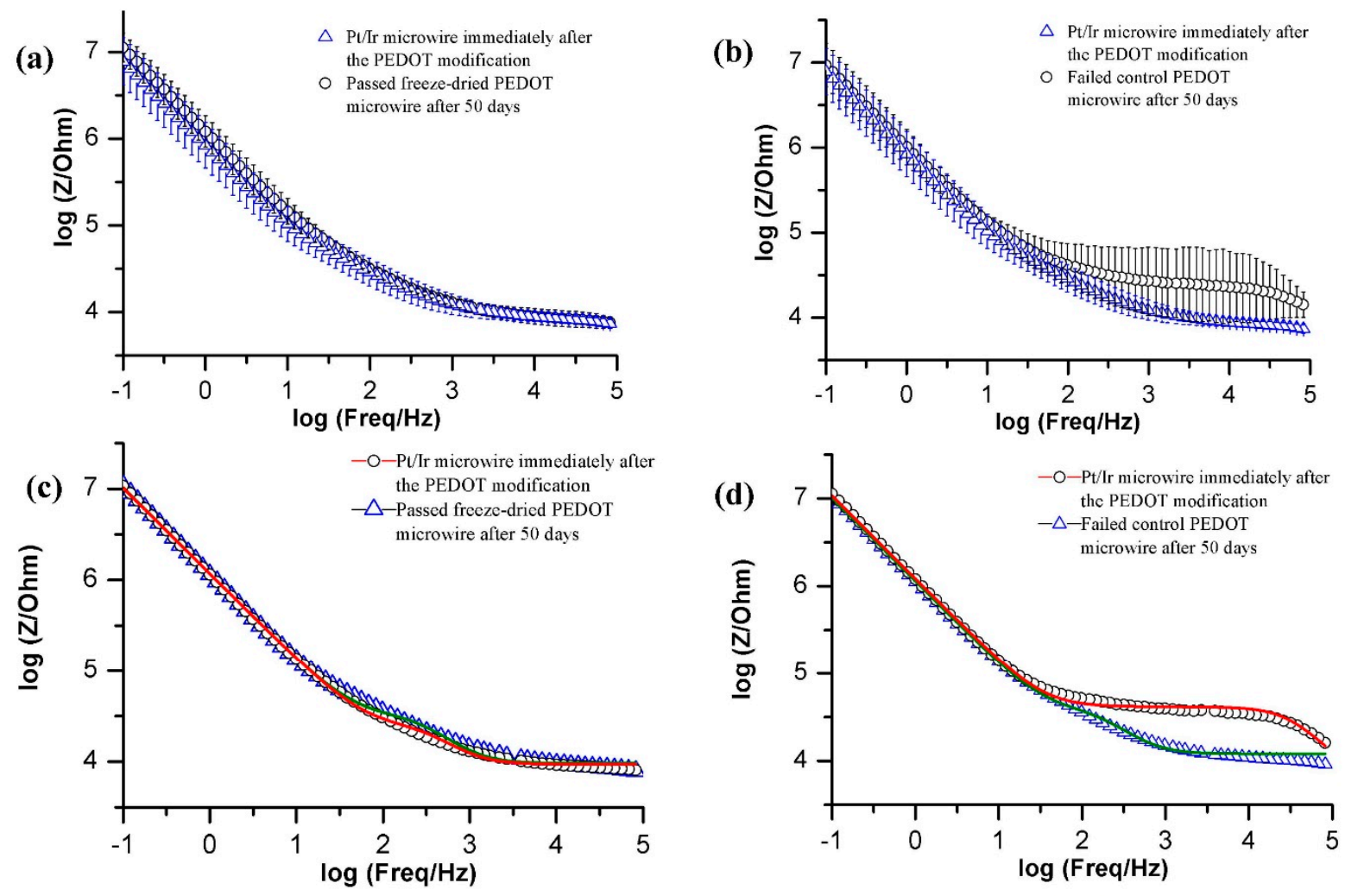

(e)

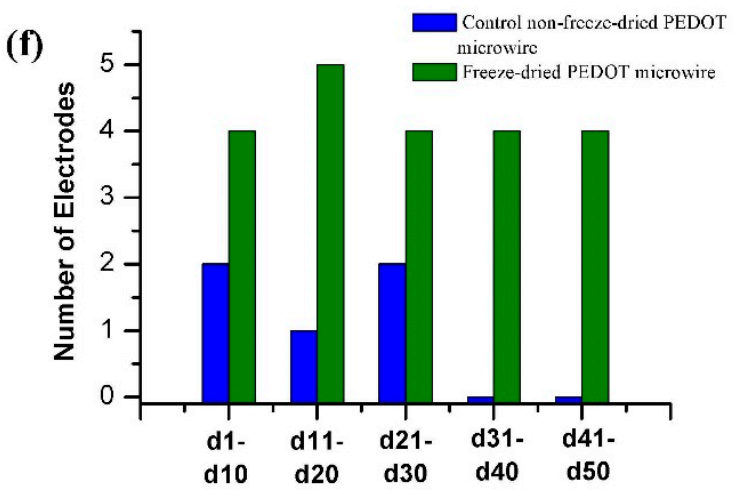

Figure 3. EIS data (with the coefficient of variation) in PBS, immediately after the PEDOTPSS modification (blue triangle) and after 50 days (black circle) of: (a) passed freeze-dried microwires; (b) control failed microwires; (c,d) EIS data from one representative microwire from each group. The red and green solid lines in both figures indicate the respective fit; (e) The circuit used to fit the experimental EIS data; (f) Stability comparison of freeze-dried $v s$. control non-freeze-dried samples. Figure shows the number of passed microwires with less than $10 \%$ change in their original impedance at $1 \mathrm{kHz}$. The green and blue bars represent the passed freeze-dried and control microwires, respectively, out of 5 in each group. 
Table 1. Fit parameters for the EIS data from a non-freeze-dried microwire immediately after the PEDOT deposition and after failing during d41-d50. Values in the parentheses correspond to the rel. std. error (\%). Parameters for a passed freeze-dried sample (d41-d50) are also added for comparison. $n$ is the corresponding frequency power necessary for a better fit for non-ideal capacitors.

\begin{tabular}{|c|c|c|c|c|}
\hline & $\begin{array}{l}\text { Non-Freeze-Dried, } \\
\text { Immediately after } \\
\text { PEDOT Modification }\end{array}$ & $\begin{array}{c}\text { Non-Freeze-Dried } \\
\text { (d41-d50) }\end{array}$ & $\begin{array}{c}\text { Freeze-Dried, } \\
\text { Immediately after } \\
\text { PEDOT Modification }\end{array}$ & Freeze-Dried (d1-d50) \\
\hline $\mathrm{R}_{\mathrm{s}}\left(\mathrm{Ohm}-\mathrm{cm}^{2}\right)$ & $\begin{array}{c}1.21 \times 10^{4} \\
\left(5.22 \times 10^{0}\right) \\
\end{array}$ & $4.31 \times 10^{3}\left(2.54 \times 10^{0}\right)$ & $\begin{array}{c}9.66 \times 10^{3} \\
\left(2.93 \times 10^{0}\right) \\
\end{array}$ & $9.39 \times 10^{3}\left(2.30 \times 10^{0}\right)$ \\
\hline $\mathrm{C}_{\mathrm{d}}\left(\mathrm{F} / \mathrm{cm}^{2}\right)$ & $\begin{array}{l}2.88 \times 10^{-8} \\
\left(1.50 \times 10^{1}\right) \\
\end{array}$ & $1.41 \times 10^{-10}\left(7.62 \times 10^{0}\right)$ & $\begin{array}{l}2.11 \times 10^{-8} \\
\left(7.70 \times 10^{0}\right) \\
\end{array}$ & $2.46 \times 10^{-8}\left(7.17 \times 10^{0}\right)$ \\
\hline $\mathrm{R}_{\text {poly }}\left(\mathrm{Ohm}-\mathrm{cm}^{2}\right)$ & $\begin{array}{l}3.75 \times 10^{4} \\
\left(2.09 \times 10^{1}\right) \\
\end{array}$ & $3.71 \times 10^{4}\left(3.15 \times 10^{0}\right)$ & $\begin{array}{l}2.96 \times 10^{4} \\
\left(9.03 \times 10^{0}\right) \\
\end{array}$ & $2.25 \times 10^{4}\left(8.89 \times 10^{0}\right)$ \\
\hline$Q_{\text {poly }}\left(\mathrm{S}-\mathrm{sec}^{\mathrm{n}} / \mathrm{cm}^{2}\right)$ & $\begin{array}{l}1.24 \times 10^{-7} \\
\left(9.49 \times 10^{0}\right)\end{array}$ & $1.44 \times 10^{-7}\left(2.69 \times 10^{0}\right)$ & $\begin{array}{l}1.28 \times 10^{-7} \\
\left(4.53 \times 10^{0}\right)\end{array}$ & $1.27 \times 10^{-7}\left(3.78 \times 10^{0}\right)$ \\
\hline$n(0<n<1)$ & $\begin{array}{l}9.38 \times 10^{-1} \\
\left(2.88 \times 10^{0}\right)\end{array}$ & $9.48 \times 10^{-1}\left(8.34 \times 10^{-1}\right)$ & $\begin{array}{l}9.38 \times 10^{-1} \\
\left(1.36 \times 10^{0}\right)\end{array}$ & $9.29 \times 10^{-1}\left(1.07 \times 10^{0}\right)$ \\
\hline
\end{tabular}

The most likely explanation for the higher stability of the freeze-dried PEDOT modified electrodes is that the freeze-drying process involves a quick initial transition to a frozen solid state and then evaporation of the solvent molecules directly from the solid to a vapor state. This process could essentially bypass the liquid phase related capillary-driven collapse of the porous PEDOT structure. In other words, freeze-drying locks the native structure of the polymer with the high electrochemically active surface area.

\section{Conclusions}

In conclusion, we showed that freeze drying could be a useful method to increase the stability of PEDOT modified neural electrodes. We also demonstrated that the probable mechanism for the failure mode is related to the collapse of the porous polymeric structure, closing of the small pores first and consequent reduction in the electrochemically active surface area. We believe that these findings will facilitate a wider use of CP modified neural electrodes by improving the shelf-life for storage and transportation, and potentially reducing the cost through repeated and long term multiple use. Besides, this may also help other fields where conductive PEDOT films are extensively used, for example, in organic electronics.

\section{Acknowledgments}

This work was sponsored by the Defense Advanced Research Projects Agency (DARPA) MTO under the auspices of Jack Judy through the Space and Naval Warfare Systems Center, Pacific Grant/Contract No. N66001-12-1-4026. 


\section{Author Contributions}

Himadri S. Mandal modified the neural probes with conductive polymers, initiated the study, analyzed the data and wrote the paper; Richard O. Cliff did the freeze-drying; Joseph J. Pancrazio organized and led the project.

\section{Conflicts of Interest}

The authors declare no conflict of interest.

\section{References}

1. Ludwig, K.A.; Langhals, N.B.; Joseph, M.D.; Richardson-Burns, S.M.; Hendricks, J.L.; Kipke, D.R. Poly(3,4-ethylenedioxythiophene) (PEDOT) polymer coatings facilitate smaller neural recording electrodes. J. Neural Eng. 2011, 8, doi:10.1088/1741-2560/8/1/014001.

2. Ludwig, K.A.; Uram, J.D.; Yang, J.; Martin, D.C.; Kipke, D.R. Chronic neural recordings using silicon microelectrode arrays electrochemically deposited with a poly(3,4-ethylenedioxythiophene) (PEDOT) film. J. Neural Eng. 2006, 3, 59-70.

3. Cui, X.T.; Zhou, D.D. Poly(3,4-Ethylenedioxythiophene) for chronic neural stimulation. IEEE Trans. Neural Syst. Rehabil. Eng. 2007, 15, 502-508.

4. Green, R.A.; Hassarati, R.T.; Bouchinet, L.; Lee, C.S.; Cheong, G.L.M.; Yu, J.F.; Dodds, C.W.; Suaning, G.J.; Poole-Warren, L.A.; Lovell, N.H. Substrate dependent stability of conducting polymer coatings on medical electrodes. Biomaterials 2012, 33, 5875-5886.

5. Gerwig, R.; Stett, A.; Stelzle, M. PEDOT-CNT composite microelectrodes for recording and electrostimulation applications: Fabrication, morphology, and electrical properties. Front. Neuroeng. 2012, 5, doi:10.3389/fneng.2012.00008.

6. Luo, X.; Weaver, C.L.; Zhou, D.D.; Greenberg, R.; Cui, X.T. Highly stable carbon nanotube doped poly(3,4-ethylenedioxythiophene) for chronic neural stimulation. Biomaterials 2011, 32, 5551-5557.

7. Mandal, H.S.; Knaack, G.L.; Charkhkar, H.; McHail, D.G.; Kastee, J.S.; Dumas, T.C.; Peixoto, N.; Rubinson, J.F.; Pancrazio, J.J. Improving the performance of poly(3,4-ethylenedioxythiophene) for brain-machine interface applications. Acta Biomater. 2014, 10, 2446-2454.

8. Mandal, H.S.; Kastee, J.S.; McHail, D.G.; Rubinson, J.F.; Pancrazio, J.J.; Dumas, T.C. Improved poly(3,4-ethylenedioxythiophene) (PEDOT) for neural stimulation. Neuromodulation 2015, doi:10.1111/ner.12285.

9. Crispin, X.; Jakobsson, F.L.E.; Crispin, A.; Grim, P.C.M.; Andersson, P.; Volodin, A.; van Haesendonck, C.; Van der Auweraer, M.; Salaneck, W.R.; Berggren, M. The origin of the high conductivity of poly(3,4-ethylenedioxythiophene)-poly(styrenesulfonate) (PEDOT-PSS) plastic electrodes. Chem. Mater. 2006, 18, 4354-4360.

10. Kayinamura, Y.P.; Ovadia, M.; Zavitz, D.; Rubinson, J.F. Investigation of near ohmic behavior for poly(3,4-ethylenedioxythiophene): A model consistent with systematic variations in polymerization conditions. ACS Appl. Mater. Interfaces 2010, 2, 2653-2662. 
11. Kozai, T.D.Y.; Langhals, N.B.; Patel, P.R.; Deng, X.; Zhang, H.; Smith, K.L.; Lahann, J.; Kotov, N.A.; Kipke, D.R. Ultrasmall implantable composite microelectrodes with bioactive surfaces for chronic neural interfaces. Nat. Mater. 2012, 11, 1065-1073.

12. Cui, X.; Martin, D.C. Electrochemical deposition and characterization of poly(3,4ethylenedioxythiophene) on neural microelectrode arrays. Sens. Actuators B Chem. 2003, 89, 92-102.

13. Collazos-Castro, J.E.; Hernández-Labrado, G.R.; Polo, J.L.; García-Rama, C. N-Cadherin- and L1-functionalised conducting polymers for synergistic stimulation and guidance of neural cell growth. Biomaterials 2013, 34, 3603-3617.

14. Yamato, H.; Ohwa, M.; Wernet, W. Stability of polypyrrole and poly(3,4-ethylenedioxythiophene) for biosensor application. J. Electroanal. Chem. 1995, 397, 163-170.

15. Chakrapani, N.; Wei, B.; Carrillo, A.; Ajayan, P.M.; Kane, R.S. Capillarity-driven assembly of two-dimensional cellular carbon nanotube foams. Proc. Natl. Acad. Sci. 2004, 101, 4009-4012.

16. Qu, J.; Zhao, Z.; Wang, X.; Qiu, J. Tailoring of three-dimensional carbon nanotube architectures by coupling capillarity-induced assembly with multiple CVD growth. J. Mater. Chem. 2011, 21, 5967-5971.

17. Liu, H.; Li, S.; Zhai, J.; Li, H.; Zheng, Q.; Jiang, L.; Zhu, D. Self-assembly of large-scale micropatterns on aligned carbon nanotube films. Angew. Chem. Int. Ed. 2004, 43, 1146-1149.

18. Futaba, D.N.; Hata, K.; Yamada, T.; Hiraoka, T.; Hayamizu, Y.; Kakudate, Y.; Tanaike, O.; Hatori, H.; Yumura, M.; Iijima, S. Shape-engineerable and highly densely packed single-walled carbon nanotubes and their application as super-capacitor electrodes. Nat. Mater. 2006, 5, 987-994.

19. Varshosaz, J.; Eskandari, S.; Tabbakhian, M. Freeze-drying of nanostructure lipid carriers by different carbohydrate polymers used as cryoprotectants. Carbohydr. Polym. 2012, 88, 1157-1163.

20. Xia, L.-W.; Xie, R.; Ju, X.-J.; Wang, W.; Chen, Q.; Chu, L.-Y. Nano-structured smart hydrogels with rapid response and high elasticity. Nat. Commun. 2013, 4, doi:10.1038/ncomms3226.

21. Moral-Vico, J.; Carretero, N.M.; Pérez, E.; Suñol, C.; Lichtenstein, M.; Casañ-Pastor, N. Dynamic electrodeposition of aminoacid-polypyrrole on aminoacid-PEDOT substrates: Conducting polymer bilayers as electrodes in neural systems. Electrochim. Acta 2013, 111, 250-260.

22. Zhou, H.; Cheng, X.; Rao, L.; Li, T.; Duan, Y.Y. Poly(3,4-ethylenedioxythiophene)/multiwall carbon nanotube composite coatings for improving the stability of microelectrodes in neural prostheses applications. Acta Biomater. 2013, 9, 6439-6449.

23. Cui, X.; Lee, V.A.; Raphael, Y.; Wiler, J.A.; Hetke, J.F.; Anderson, D.J.; Martin, D.C. Surface modification of neural recording electrodes with conducting polymer/biomolecule blends. J. Biomed. Mater. Res. 2001, 56, 261-272.

24. Cui, X.; Hetke, J.F.; Wiler, J.A.; Anderson, D.J.; Martin, D.C. Electrochemical deposition and characterization of conducting polymer polypyrrole/PSS on multichannel neural probes. Sens. Actuators A Phys. 2001, 93, 8-18.

(C) 2015 by the authors; licensee MDPI, Basel, Switzerland. This article is an open access article distributed under the terms and conditions of the Creative Commons Attribution license (http://creativecommons.org/licenses/by/4.0/). 